\title{
The Effect of Selective Brainstem or Spinal Cord Lesions on Treadmill Locomotion Evoked by Stimulation of the Mesencephalic or Pontomedullary Locomotor Regions
}

\author{
B. R. Noga, D. J. Kriellaars, ${ }^{a}$ and L. M. Jordan \\ Department of Physiology, Faculty of Medicine, University of Manitoba, Winnipeg, Canada R3E OW3
}

The descending pathways from the brainstem locomotor areas were investigated by utilizing reversible cooling (to block synaptic or fiber transmission) and irreversible subtotal lesions of the brainstem or spinal cord (C2-C3 level). Experiments were conducted on decerebrate cats induced to walk on a treadmill by electrical stimulation of the brainstem. Locomotion produced by stimulation of the mesencephalic locomotor region (MLR) was not abolished by caudal brainstem lesions that isolated the lateral tegmentum or by extended rostral/caudal dorsal hemisections of the spinal cord. These results demonstrate that the MLR does not require a pathway projecting through the lateral tegmentum of the brainstem or the dorsal half of the spinal cord, as previously suggested (Mori et al., 1977, 1978b; Shik and Yagodnitsyn, 1978; Shik, 1983). Rather, the results indicate that the descending pathway originating from the MLR projects through the medial reticular formation (MedRF) and the ventral half of the spinal cord. Locomotion produced by stimulation of the pontomedullary locomotor region (PLR) was blocked by reversible cooling of either the MedRF or the ventrolateral funiculus of the spinal cord. In some cases, locomotion could be produced by stimulation of the PLR following extended dorsal hemisections of the spinal cord. These results demonstrate that the PLR can also produce locomotion by activation of cells in the MedRF that project caudally through the ventral half of the spinal cord. Stimulation of the PLR could also elicit locomotion following its surgical isolation from the MedRF of the brainstem. Furthermore, lesions of the dorsal spinal cord resulted in the loss of PLR-evoked locomotion in some, but not all, cases. Thus, an alternative projection of the PLR through the dorsal half of the spinal cord (Kazennikov et al., 1980, 1983a,b; Shik, 1983) cannot be ruled out. Overall, these results demonstrate that the PLR is not an essential component of the motor pathway originating from the MLR. The organizational scheme of "brainstem locomotor regions" is discussed in the context of recent information demonstrating a link be-

\footnotetext{
Received June 20, 1990; revised Nov. 28, 1990; accepted Jan. 11, 1991.

We would like to thank Dr. R. Brownstone, Dr. B. Mallory, G. Brown, and D. DeJong for their technical assistance during some of the experiments. This research was supported by a Medical Research Council of Canada (MRC) grant to L.M.J.

Correspondence should be addressed to Dr. B. R. Noga, Department of Physiology, Faculty of Medicine, University of Manitoba, Winnipeg, Manitoba, Canada R3E 0W3.

a Present address: School of Medical Rehabilitation, 311 Rehab Center, University of Manitoba, Winnipeg, Manitoba, Canada R3E 0W3.

Copyright (C) 1991 Society for Neuroscience 0270-6474/91/111691-10\$03.00/0
}

tween the sensory component of the trigeminal system and locomotor pathways (Noga et al., 1988).

Stimulation of the mesencephalic locomotor region (MLR; Shik et al., 1966, 1967) within the caudal cuneiform nucleus of the midbrain (stereotaxic coordinates: P2, L4, H-1; Berman, 1968) produces locomotion in decerebrate cats placed on a treadmill. This site has been implicated in mediating the effects of higher motor centers (Shik et al., 1968; Garcia-Rill, 1986) for the activation of the spinal locomotor system (cf. Jordan, 1986). It is thus important to establish the descending trajectory of this area. The MLR does not project directly to the spinal cord (Orlovsky, 1969, 1970; Steeves and Jordan, 1984; Garcia-Rill, 1986), and two potential relay sites within the brainstem have previously been described (Noga et al., 1988) using the technique of localized injection of neuroactive substances.

The first site has been localized to the pontomedullary medial reticular formation (MedRF). On the basis of the available anatomical and electrophysiological evidence (cf. Jordan, 1986; Garcia-Rill and Skinner, 1987; Noga et al., 1988), it is thought that reticulospinal cells originating in the MedRF project caudally via the ventrolateral funiculus (VLF) of the spinal cord. This supports the observation that the VLF is important for MLR-evoked locomotion (Steeves and Jordan, 1980) or for locomotion in otherwise intact animals, including man (Afelt, 1974; Afelt et al., 1975; Eidelberg, 1980, 1981; Eidelberg et al., 1981a,b; Sholomenko and Steeves, 1987).

The second site has been localized to the lateral tegmentum of the brainstem and corresponds to the area known as the pontomedullary locomotor region (PLR) previously shown to produce locomotion in decerebrate cats (Mori et al., 1977, 1978a,b; Shik and Yagodnitsyn, 1977, 1978; Kazennikov et al., 1979, 1983a; Budakova and Shik, 1980; Selionov and Shik, 1981, 1984). On the basis of electrophysiological recording techniques, it has been suggested that the PLR extends throughout the brainstem as a polysynaptic pathway, with the cells of origin (the pontomedullary locomotor column) distributed either within (Shik and Yagodnitsyn, 1978; Selionov and Shik, 1984) or medial and ventral (Selionov and Shik, 1984) to its fiber tract [the pontomedullary locomotor strip (PLS)]. The polysynaptic pathway is thought to continue through the dorsal aspect of the spinal cord with the cells of origin located primarily in the dorsal horn and their fibers located in the dorsolateral funiculus (DLF) at the cervical level (Mori et al., 1977; Kazennikov et al., 1980, 1983a, 1985; Shik, 1983).

Evidence has been presented in favor of the idea that the MLR projects via the PLR (Mori et al., 1977, 1978b; Shik and 
Yagodnitsyn, 1978; Shik, 1983). However, this is inconsistent with the existing anatomical data (Steeves and Jordan, 1984; Bayev et al., 1988), which demonstrate that the predominant descending projection of the MLR is to the MedRF. Furthermore, whereas locomotion produced by stimulation of the MLR is reversibly abolished during cooling of the MedRF to temperatures that block synaptic transmission, it is unaffected by cooling of the PLR (Shefchyk et al., 1984).

An alternative interpretation has been proposed by Noga et al..(1988), who suggested that the PLR is part of the trigeminal system involved in sensory activation of locomotion, an idea that finds support in the findings of Aoki and Mori (1981) and the more recent studies by Bayev and colleagues (Bayev et al., 1988; Beresovskii and Bayev, 1988). Based upon this interpretation of brainstem pathways involved in locomotion, one would expect that the MLR would not require the integrity of the lateral tegmental pathway in order to produce locomotion when electrically stimulated. Furthermore, one would expect that the PLR could produce locomotion by activation of pathways projecting either to the MedRF or directly to the spinal cord (or some combination of both) since trigeminoreticular and trigeminospinal projections have been demonstratcd both anatomically and electrophysiologically (Torvik, 1956; Lamarche et al., 1960; Stewart and King, 1963; Nord and Ross, 1973; Matsushita et al., 1981, 1982).

Experiments were therefore designed to determine the descending trajectories of both the MLR and the PLR by examining the effect of reversible (cooling) and irreversible lesions of the brainstem and the spinal cord on locomotion produced by stimulation of either the MLR or the PLR.

Preliminary data have been presented elsewhere (Noga et al., 1986; Noga, 1988).

\section{Materials and Methods}

The experiments were carried out on 15 adult cats weighing $2.5-4.1 \mathrm{~kg}$. Animals were initially anesthetized with a mixture of nitrous oxide and halothane. The trachea was intubated, the left common carotid was cannulated, and blood pressure was monitored with a pressure transducer. Each animal was given $4 \mathrm{mg}$ dexamethasone (Hexadrol phosphate, Organon) intravenously to reduce tissue swelling. The head of each animal was fixed in a stereotaxic headframe with all four limbs free to step on a treadmill belt. The hindquarters were suspended by a sling under the abdomen. The animals were decerebrated with a precollicular-postmamillary transection of the brainstem, and the anesthesia was subsequently terminated. All wound margins were anesthetized with topically applied Xylocaine ointment or by small injections of Xylocaine hydrochloride. Following a recovery period of approximately $1-1.5 \mathrm{hr}$, the cerebellum and brainstem were exposed by an extended craniotomy. In some experiments the cervical spinal cord was exposed by removal of the $\mathrm{C} 2$ or $\mathrm{C} 3$ vertebral laminae.

In order to establish the descending trajectories of the MLR and PLR, electrical stimulation was applied to these sites to produce locomotion both before and after irreversible lesions or reversible cooling of the brainstem or spinal cord. Because the animals were suspended over the treadmill with their limbs free to move, various patterns of stepping such as quadrupedal or bipedal (forelimb or hindlimb) locomotion, commonly seen with stimulation of the brainstem locomotor regions (Shik et al., 1966, 1967; Shik and Yagodnitsyn, 1977, 1978; Mori et al., 1978a,b; Amemiya and Yamaguchi, 1984; Ross and Sinnamon, 1984; Noga et al., 1988) could be assessed. Stimulating electrodes were stereotaxically positioned in the MLR and PLR regions of the brainstem following the decerebration and recovery period. Control bouts of locomotion of 10-30-sec duration were recorded following electrical stimulation of the MLR and PLR (square-wave pulse, 0.5-1.0-msec duration, $20-40 \mathrm{~Hz}, 10-180 \mu \mathrm{A}$ ). All stimulation parameters were noted, including the electrical threshold for the initiation of locomotion (determined by slowly increasing the strength of stimulation until locomotion ensues). Locomotion was monitored using electromyographic
(EMG) electrodes placed bilaterally in various muscles of the forelimbs or hindlimbs: lateral gastrocnemius (LG); tibialis anterior (TA); triceps brachii (TB), and biceps brachii (BB). The EMG signals were amplified and recorded using an 8-track FM analog magnetic tape recorder (bandpass, $10 \mathrm{~Hz}$ to $2.25 \mathrm{kHz}$ ) for later analysis. Angular displacement of the limbs in the parasagittal plane was qualitatively assessed by visual inspection during controlled bouts of locomotion. Only gross changes in limb angular displacement (approximately $50 \%$ or more) following the brainstem or spinal cord lesions are reported.

Reversible lesions of the brainstem were made by cooling a localized area with a coaxial 18-gauge stainless-steel probe through the inner shaft of which cooled alcohol was circulated. Cooling probe temperatures were monitored by a tissue-implantable thermocouple microprobe (type IT-23, Bailey Instruments) glued to the outside surface of the probe tip. Probe-tip temperatures were kept at levels that will effectively block synaptic $\left(18-20^{\circ} \mathrm{C}\right)$ but not axonal $\left(4-10^{\circ} \mathrm{C}\right)$ transmission (Brooks, 1983). Probe-tip temperatures of $13-15^{\circ} \mathrm{C}$ were sufficient to block synaptic transmission in the hrainstem for distances of 1-2 $\mathrm{mm}$ from the side of the probe. The temperature of the region was allowed to return to the control level by stopping the flow of cold alcohol. Reversible lesions of various areas of the cervical spinal cord werc obtained by cooling (intradurally) the surface of the spinal cord with a U-shaped cooling probe through which cooled alcohol was circulated. Probe-tip temperatures were kept at levels that would effectively block axonal transmission (Brooks, 1983) in the immediately adjacent spinal cord quadrant. At these probe-tip temperatures, synaptic transmission throughout the spinal cord segment at which cooling was performed was also blocked. Isotherm measurements of the spinal cord during intradural cooling of the VLF revealed that fiber transmission in the adjacent spinal quadrant would begin to block at probe-tip temperatures of $8-10^{\circ} \mathrm{C}$. Complete blockage of fiber transmission in the adjacent spinal quadrant (for distances of 1-1.5 mm from the probe tip) was observed at probe-tip temperatures of $4-8^{\circ} \mathrm{C}$.

Irreversible brainstem and spinal cord $(\mathrm{C} 2-\mathrm{C} 3)$ lesions were made using surgical instruments. To prevent movement, the animals were temporarily paralyzed with an intravenous injection $(0.5-1.0 \mathrm{ml}$ of 20 $\mathrm{mg} / \mathrm{ml}$ ) of succinylcholine chloride (Quelicin chloride). During this procedure the animals were artificially respirated. The spinal cord was cooled prior to the lesion by covering the dorsal and lateral surfaces with frozen artificial cerebrospinal fluid. Once neuromuscular transmission was restored (usually 10-20 min), the artificial ventilation was discontinued. Intravenous injection of diluted norepinephrine was given temporarily in some cases to maintain blood pressure at nominal control values.

The locations of the brainstem stimulus sites were marked electrolytically (2-5 mA for 3-5 sec) at the end of each experiment. The brainstems were then removed and placed in Lillie's neutral buffered formalin overnight. Frozen sections $(30 \mu \mathrm{m})$ were cut and stained according to the method of Klüver and Barrera (1953). The location of the stimulus sites and the location and extent of the brainstem and spinal cord lesions were verified histologically.

Periods of EMG recordings, 10-20 sec in duration, were full-wave rectified, low-pass filtered, and analyzed with a Masscomp MC563 computer at the end of each experiment. The EMG linear envelope signals from all muscles were digitized at $200 \mathrm{~Hz}$ using a 1-MHz 16-channel analog-digital (A/D) converter and stored on computer hard disk. The signals were then plotted for visual inspection of the timing and amplitude of the EMG signals during the step cycle.

\section{Results}

\section{Spinal cord lesions}

Two basic results were obtained from experiments in which the spinal cord was lesioned at the C2-C3 level: (1) the MLR does not require the continuity of the dorsal half of the spinal cord, and (2) the PLR may produce locomotion in the absence of the dorsal half of the spinal cord but also shows a loss in the capacity to produce locomotion with these spinal lesions more often than that seen with MLR stimulation. These results are presented in Figures 1-3.

Effects on $M L R$-evoked locomotion. Figure 1 shows the effect of an extended (8- $\mathrm{mm})$ dorsal hemisection at the C2-C3 level on MLR-evoked locomotion. Following this extensive lesion, 


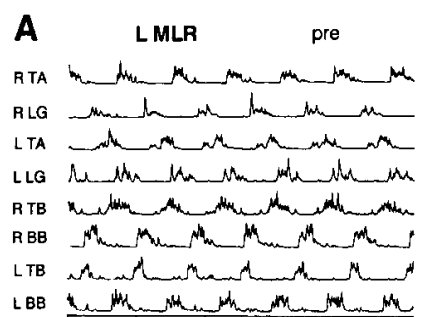

B

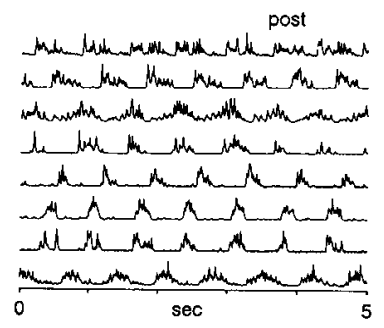

E

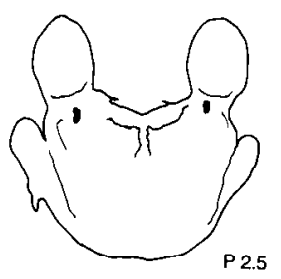

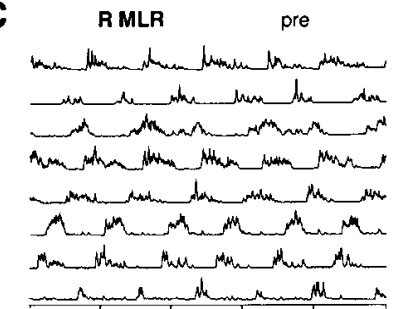

D

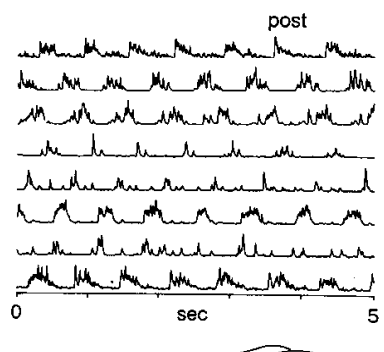

F

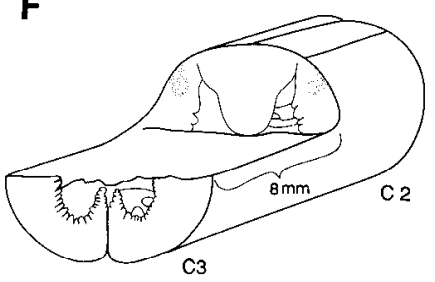

Figure 1. MLR-evoked locomotion does not require the integrity of the dorsal spinal cord. $A$ and $C$ illustrate control runs of left- and rightMLR-evoked quadrupedal treadmill locomotion, respectively. Following a $\mathrm{C} 2-\mathrm{C} 3$ dorsal hemisection $(F)$, left and right MLR sites (solid lesion sites in $E$ ) maintain their ability to produce locomotion ( $B$ and $D$, respectively) and at similar stimulation parameters. The spinal cord surface shown at the C3 level indicates the maximum extent of the lesion determined histologically. The lesion spanned $8 \mathrm{~mm}$ in total length. Stippled regions in $F$ indicate areas of PLS (Kazennikov et al., 1983b). MLR stimulation parameters were $40 \mathrm{~Hz}, 0.5$-msec duration (all trials); $175 \mu \mathrm{A}(A$ and $C), 150 \mu \mathrm{A}(B)$, and $200 \mu \mathrm{A}(D)$. EMG recordings in $B-D$ are in same order as listed in $A . P$ levels in this and following figures indicate posterior stereotaxic planes in millimeters from the interaural line (Berman, 1968). $L$, left; $R$, right; pre, before spinal lesion; post, following spinal lesion.

stimulation of the MLR (either side) could still produce quadrupedal locomotion that was essentially the same as that seen in the control situation. No change in the threshold for the production of locomotion by electrical stimulation of the MLR was observed.

Figure 3 summarizes the effect of various spinal cord lesions on locomotion produced by stimulation of the MLR. In general, stimulation of the MLR could produce locomotion virtually identical to that seen in the control situation following disruption of the DLF, dorsal horns, or the dorsal columns of the spinal cord by either simple transections (Fig. $3 A, B$ ) or extended lesions (Fig. 3D,F,I). This was true for 5 of 7 experimental animals and 9 of 12 tested MLR stimulation sites. No consistent difference in the threshold for the production of locomotion by electrical stimulation of the MLR was observed in these experiments (cf. Fig. 1). Lesions of the dorsal spinal cord diminished the capacity of 3 of 12 MLR stimulation sites to produce locomotion similar to the control locomotion trials (in 2 of 7 experiments) but never abolished locomotion entirely (Fig. $3 G, H)$. These changes were seen either as a complete loss or as a decrease in the angular displacement (paralleled by diminished amplitude of the phasic EMG responses) of the affected limbs. In one experiment (Fig. 3C), partial transection of the VLF

ipsilateral to the MLR stimulation site resulted in the loss of ipsilateral hindlimb locomotion and a noticeable decrease in the angular displacement of the ipsilateral forelimb and contralateral hindlimb during the swing and stance phases. This latter effect was also apparent as a decrease in the amplitude of the EMG signals recorded from each of the affected limbs.

Effects on PLR-evoked locomotion. Figure 2 shows the effect of an extended dorsal hemisection at the C2-C3 level on PLRevoked locomotion (lesion shown in Fig. 1). In this experiment, stimulation of the left or right PLR was capable of producing bouts of hindlimb or forelimb locomotion, respectively. No consistent differences in the threshold for the production of this locomotion were observed. However, in both cases, the locomotion observed following the lesion was bipedal compared to the quadrupedal control situation. Interestingly, stimulation of the right PLR following the dorsal hemisection was also capable of producing right hindlimb locomotion, but this was not observed simultaneously with the bilateral forelimb locomotion.

Forelimb or hindlimb locomotion could also be produced by stimuli applied in the PLR following hemisection of extended portions (up to $8.5 \mathrm{~mm}$ ) of the dorsal aspect of the spinal cord (Fig. $3 H-J$ ) or following extended lesions of the dorsal horn (Fig. $3 D-G$ ). These results show that PLR stimulation may produce locomotion by pathways traveling in the ventral half of the spinal cord at the $\mathrm{C} 2-\mathrm{C} 3$ level. This was supported by the observation that, following partial transection of the ipsilateral VLF (Fig. 3C), stimuli applied in the ipsilateral PLR or in mul-

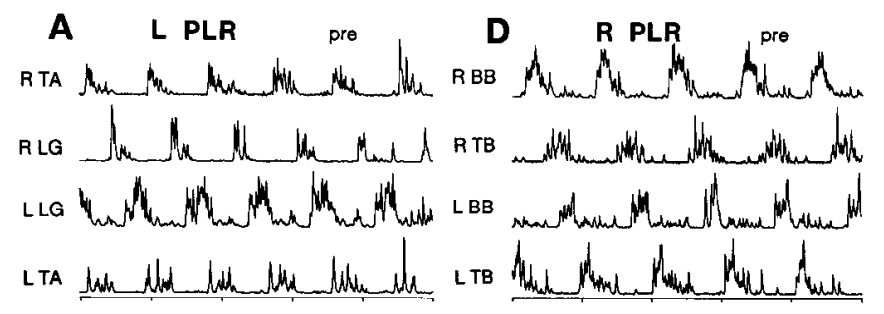

B

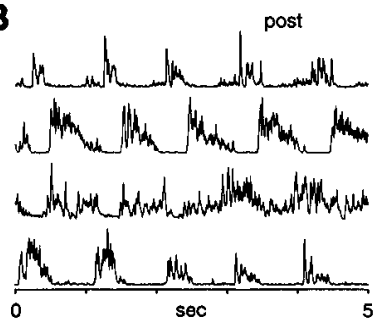

C

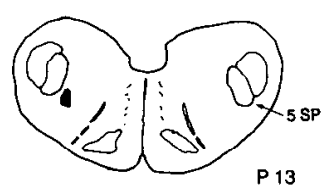

E
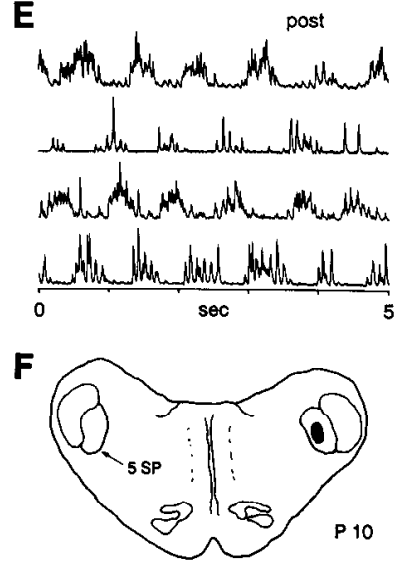

Figure 2. Stimulation of the PLR may produce locomotion following an extensive dorsal hemisection of the spinal cord at the $\mathrm{C} 2-\mathrm{C} 3$ level. $A$ and $D$ show control bouts of locomotion produced by stimulation of the left and right PLR, respectively. Following the C2-C3 dorsal hemisection indicated in $F$, the left and right PLR still maintain their ability to produce hindlimb or forelimb locomotion ( $B$ and $E$, respectively) when electrically stimulated. Stimulation sites are illustrated by solid lesion sites in $C$ and $F$. PLR stimulation parameters were $40 \mathrm{~Hz}, 0.5$ msec duration (all trials); $175 \mu \mathrm{A}(A), 135 \mu \mathrm{A}(B), 180 \mu \mathrm{A}(D)$, and 200 $\mu \mathrm{A}(E) .5 S P$ : spinal nucleus of the trigeminal nerve; $L$, left; $R$, right; pre, before spinal lesion; post, following spinal lesion. 


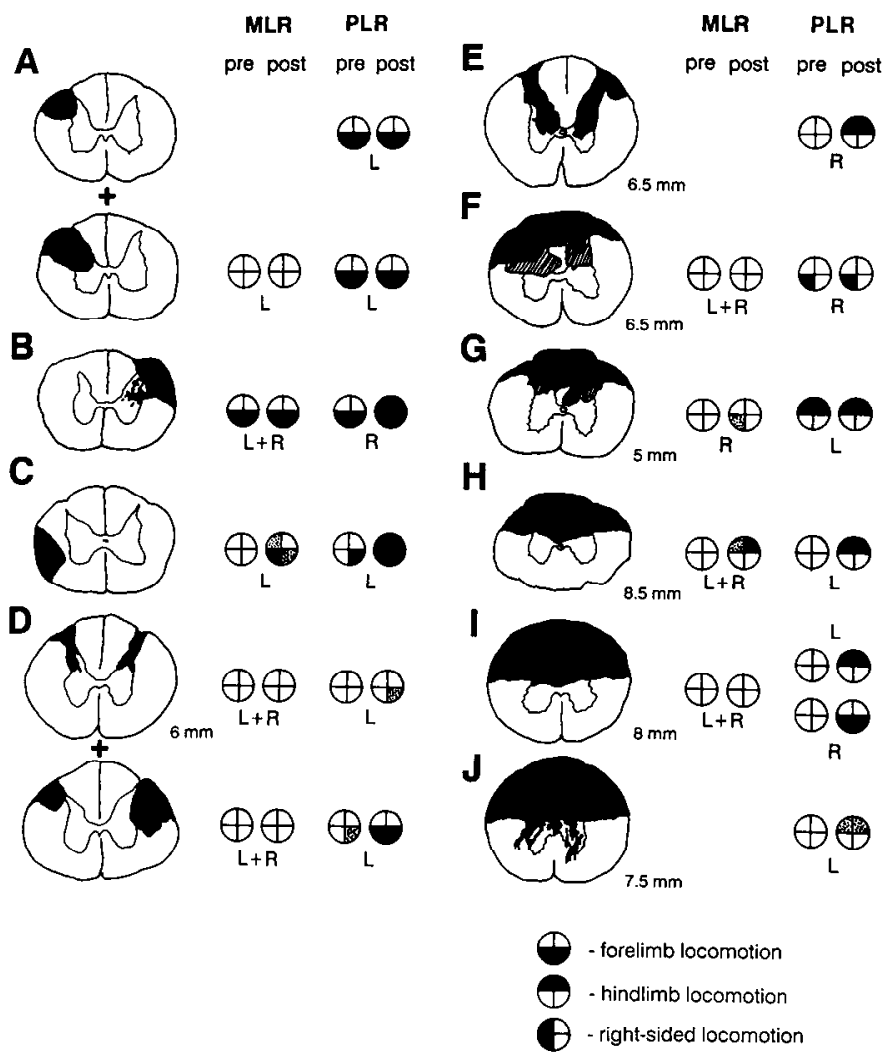

Figure 3. Summary of MLR- and PLR-evoked locomotion before and after various subtotal spinal cord lesions (darkened areas; $\mathrm{C} 2$ or $\mathrm{C} 3$ levels). Each experiment is shown separately. Lesions in $A-C$ (and $b o t-$ tom lesion in $D$ ) are simple transections. All other lesions are extended (rostral-caudal) for various indicated lengths (numbered, in millimeters). The locomotion produced by MLR or PLR stimulation before (pre) and after (post) each lesion is shown in separate columns to the right of each panel. Stimulation of the left $(L)$ or right $(R)$ MLR or PLR is also indicated. Each limb is represented by quadrants of a circle: forclimbs arc located in upper quadrants, hindlimbs in lower quadrants, and left and right limbs by the left and right halves, respectively. Limbs illustrating locomotor movements are indicated schematically by the open regions; limbs not participating in locomotion are indicated by solid quadrants. Limbs that show decreased amplitude of limb displacement and EMG activity are indicated by the stippled quadrants.

tiple locations throughout the dorsolateral tegmentum were incapable of producing locomotion (even though stimulation of the ipsilateral MLR could still produce contralateral forelimb locomotion and some ipsilateral forelimb and contralateral hindlimb locomotion). In one additional experiment (Fig. $4 F-$ $H$ ), intradural cooling of the spinal cord at the $\mathrm{C} 2$ level (over a length of $1 \mathrm{~cm}$ ) to temperatures sufficient for the block of fiber transmission in the immediately adjacent VLF and synaptic transmission throughout the $\mathrm{C} 2$ spinal cord level (probe temperatures to $6^{\circ} \mathrm{C}$ ) reversibly abolished locomotion produccd by stimulation of the ipsilateral PLR. On the other hand, cooling of the dorsal columns (Fig. 4A-C) or the DLF (Fig. $4 C-E$; to probe temperatures of $-3^{\circ} \mathrm{C}$ and $1^{\circ} \mathrm{C}$, respectively) had no observable effect on PLR-evoked locomotion.

The contribution of the dorsal aspect of the spinal cord to the production of PLR-evoked locomotion is more difficult to assess (see Discussion). Although lesions of the dorsal cord had no noticeable effect on PLR-evoked locomotion in some cases (Fig. $3 A, F, G)$, the lesions affected the capacity of the PLR to produce locomotion similar to control trials more frequently than that observed for the MLR stimulation sites (see above). This de- creased capacity was observed for 7 of 10 tested PLR stimulation sites in 6 of 9 experimental animals (Fig. $3 B, D, E, H-J$ ). Deficits were seen either as a complete loss of locomotion or as a decrease in the angular displacement (paralleled by diminished amplitude of the phasic EMG responses) of the affected limbs. Furthermore, in animals that showed locomotion in hindlimbs and forelimbs during control trials (Fig. 3D-F,H-J), lesions of the dorsal aspect of the spinal cord affected the locomotor capacity of the forelimbs slightly more often than the hindlimbs (four as opposed to two tested PLR stimulation sites, respectively).

\section{Brainstem lesions}

The spinal cord lesion experiments do not determine whether the MLR and PLR are functionally independent at the level of the brainstem. Experiments were therefore designed to deter-
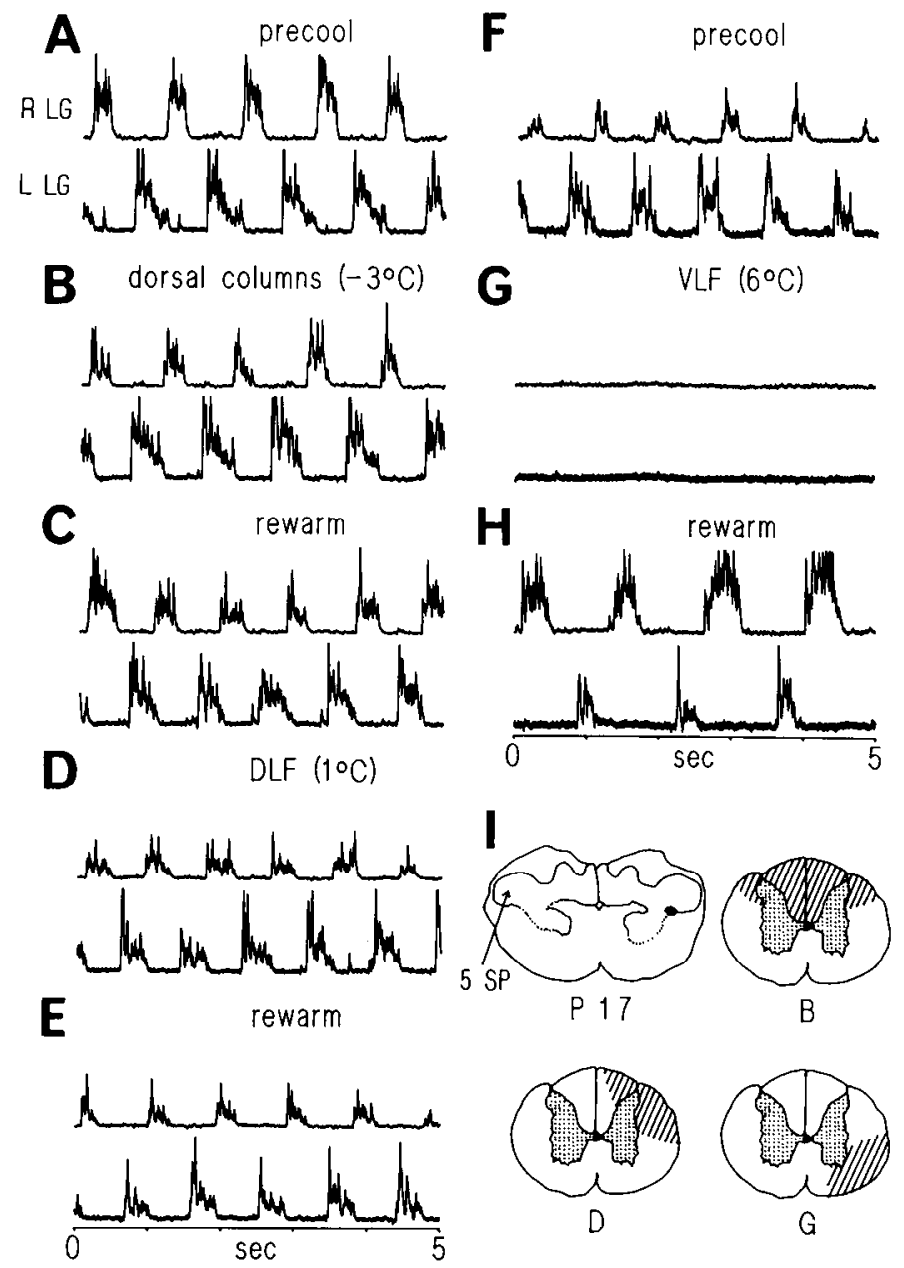

Figure 4. Effects of reversible intradural cooling of the dorsal columns, DLF, or VLF of the spinal cord (C2-C3; to temperatures sufficient to block synaptic transmission throughout the gray matter and fiber transmission in the same quadrant) on hindlimb locomotion elicited by stimulation of the ipsilateral PLR at the P17 level (solid lesion site indicated in $I) . A-C$ illustrate the responses produced by stimulation of the PLR prior to, during, and following cooling of the dorsal columns to a probe-tip temperature of $-3^{\circ} \mathrm{C}$. The effect of cooling the $\operatorname{DLF}\left(1^{\circ} \mathrm{C}\right)$ and VLF $\left(6^{\circ} \mathrm{C}\right)$ is illustrated in $D$ and $G$, respectively. The extent of liber (halched) and synaptic (stippled) transmission blockage is estimated in $I$ for each of the trials. Letters beneath spinal cord sections indicate the corresponding cooling trials in $B, D$, and $G$. PLR stimulation parameters were $30 \mathrm{~Hz}, 0.5$-msec duration (all trials); $150 \mu \mathrm{A}(A-C)$, $175 \mu \mathrm{A}(D-H)$. All EMGs are at the same gain. $5 S P$, spinal nucleus of the trigeminal nerve; $L$, left; $R$, right. 
mine the functional independence of these two brainstem sites and are summarized in Figures 5 and 6.

Effects on MLR-evoked locomotion. The effects of isolation of the lateral tegmentum of the brainstem on locomotion produced by stimulation of the MLR were examined in two experiments. The result from one of these experiments is illustrated in Figure 5, $A$ and $B$. Ipsilateral separation of the lateral tegmentum at the level of the pons and medulla (from P 4-12) did not abolish the capacity of the MLR to produce quadrupedal locomotion when electrically stimulated in either experiment. While increases in the electrical threshold for the initiation of locomotion were observed for the MLR sites (increases to 150 and $170 \mu \mathrm{A}$ from control strengths of 125 and $115 \mu \mathrm{A}$, respectively), the changes may have reflected a generalized deterioration of the preparation following the extensive lesion of the brainstem.

Effects on PLR-evoked locomotion. In order to determine if the PLR can produce locomotion without participation of the MLR and MedRF areas, the effectiveness of electrical stimuli applied to the PLR to evoke locomotion was examined following surgical isolation of the lateral tegmentum from these areas. In one experiment, stimulation of the PLR within the isolated lateral tegmental area (at the P 9 level) was still capable of producing locomotion, though the electrical threshold for the initiation of locomotion increased $90 \mu \mathrm{A}$ over the control value of $100 \mu \mathrm{A}$. In a second experiment, stimulation of the PLR within the isolated tegmental region was ineffective in initiating locomotion. However, histological examination revealed that the lesion also destroyed the main blood supply to the lateral tegmentum, and the loss of locomotor capability could have been due to the death of this region rather than to the interruption of any descending pathway from the PLR.

The results of the spinal cord lesion experiments demonstrate that the PLR can produce locomotion (forelimb or hindlimb) via pathways descending in the ventral half of the spinal cord. To determine whether a synaptic activation of the reticulospinal system originating in the MedRF could mediate locomotion produced by stimulation of the PLR, reversible cooling of the MedRF was performed. A total of five reversible MedRF cooling trials were obtained during PLR-evoked locomotion with similar results. Figure 6 demonstrates that cooling of the MedRF to temperatures sufficient for the block of synaptic transmission (probe-tip temperature, $14.7^{\circ} \mathrm{C}$ ) reversibly abolished PLR-crokcd quadrupedal locomotion. The cooling probe was displaced 1 $\mathrm{mm}$ to the contralateral side of the PLR stimulation site to ensure that, on the ipsilateral side, only synaptic transmission to cells in the MedRF was affected. Cooling of this same site also reversibly abolished locomotion elicited by MLR stimulation. The PLR site (Fig. $6 D$ ) was located 3-3.5 mm lateral to the midline, in a region slightly more medial and ventral to sites that could produce locomotion with injection of picrotoxin or substance P (Noga et al., 1988) and may therefore comprise part of the medially directed projection of the PLR on its course to the reticular formation (Selionov and Shik, 1981, 1984).

\section{Discussion}

\section{The MLR descending pathway}

The results of the present study provide additional evidence that stimulation of the MLR produces quadrupedal locomotion by activation of medially located reticulospinal cells that project to the spinal locomotor centers via a pathway located in the ventral funiculi (Fig. 7; Steeves and Jordan, 1980, 1984). This supposition is based on the observations that (1) extended spinal

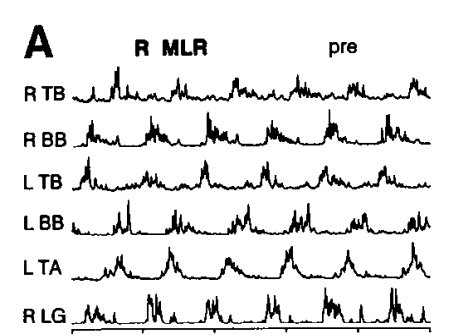

B
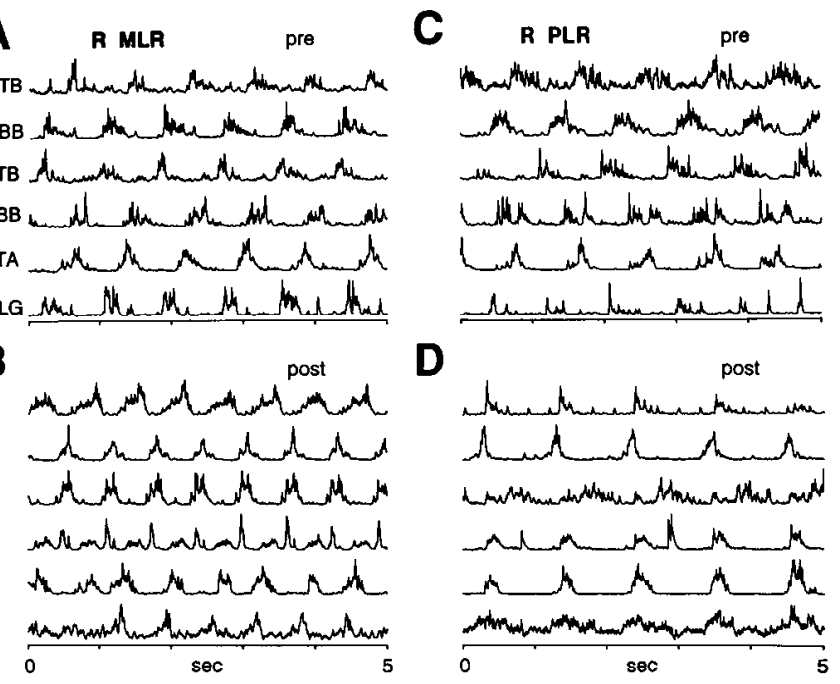

D
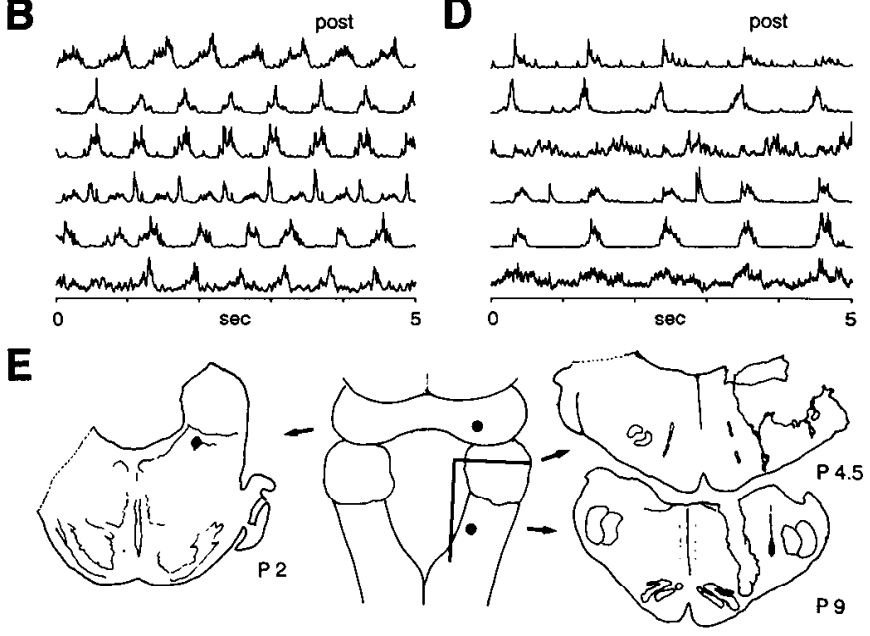

Figure 5. Isolation of the lateral portion of the brainstem does not abolish the ability of the MLR or the PLR to produce locomotion when stimulated. Locomotion was monitored using EMG recordings from both forelimb and hindlimb muscles. $A$ and $C$ show the control bouts of locomotion produced by stimulation of the right MLR $(125 \mu \mathrm{A})$ and the right PLR (100 $\mu \mathrm{A})$, respectively. Following an extensive (from $P$ 4-1 level) longitudinal lesion placed approximately $2 \mathrm{~mm}$ lateral to the midline (with a transverse section extending to the lateral edge of the brainstem at the rostral end indicated by transverse section at the $P 4.5$ level in $E$ ), stimulation of the MLR or the PLR was still capable of producing locomotion ( $B$ and $D$, respectively). The brainstem lesion is shown in $E$, as are locations of the stimulation sites (solid regions) for the MLR $(P 2)$ and the PLR $(P 9)$. Stimulation strengths for the production of locomotion following the brainstem lesion are $150 \mu \mathrm{A}(B)$ and $190 \mu \mathrm{A}(D)$. Stimulation parameters were $30 \mathrm{~Hz}, 1.0$-msec duration (all trials). $L$, left; $R$, right; pre, before brainstem lesion; post, following brainstem lesion.

dorsal hemisections or isolation of the ipsilateral tegmentum caudal to the MLR does not abolish MLR-evoked locomotion, (2) cooling of the MedRF can abolish MLR-evoked locomotion (cf. Shefchyk et al., 1984), and (3) lesions of the VLF produce deficits in locomotion evoked by stimulation of the MLR. The results thus support the original idea proposed by Orlovsky (1970) that the mediating link for the initiation of locomotion is the reticulospinal system.

The results from the present study also demonstrate that the MLR does not require a pathway projecting through the lateral tegmentum of the brainstem or the dorsal half of the spinal cord, as has been previously suggested (Mori et al., 1977, 1978b; Shik and Yagodnitsyn, 1978; Kazennikov et al., 1980, 1983a; Shik, 1983). The fact that little or no consistent changes in the electrical threshold for MLR-evoked locomotion were observed following the spinal or brainstem lesions suggests that little (if any) of the signal producing locomotion with stimulation of the MLR is transmitted through lateral brainstem pathways or the dorsal half of the spinal cord.

Although there is some evidence for a topographic relation- 


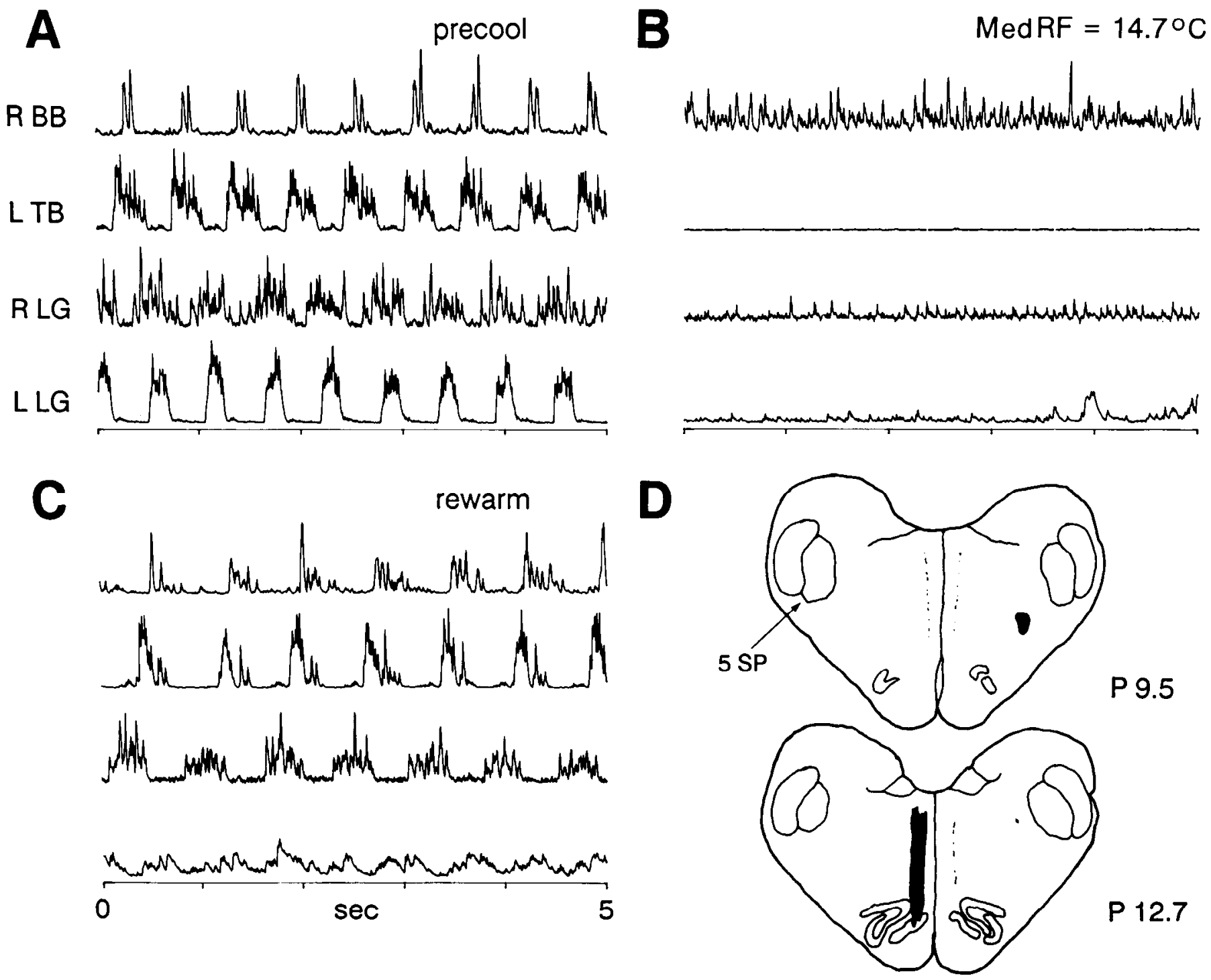

Figure 6. Cooling of the MedRF to temperatures sufficient for block of synaptic transmission can block locomotion produced by stimulation of the PLR. $A-C$ illustrate the responses produced by stimulation of the right PLR (solid lesion site indicated in $D$ at the $P 9.5$ level) prior to, during, and following local cooling of the MedRF, respectively. MedRF cooling site is also indicated by the cooling probe track (solid region) in $D$ ( $P$ 12.7). PLR stimulation parameters were $40 \mathrm{~Hz}, 0.5$-msec duration (all trials); $160 \mu \mathrm{A}(A), 200 \mu \mathrm{A}(B), 195 \mu \mathrm{A}(C)$. All EMGs are at the same gain. $5 S P$, spinal nucleus of the trigeminal nerve; $L$, left; $R$, right.

ship of forelimb- and/or hindlimb-locomotion-producing sites within the MLR (Mori et al., 1982; Amemiya and Yamaguchi, 1984), the MedRF (Drew and Rossignol, 1984; Ross and Sinnamon, 1984; Garcia-Rill and Skinner, 1987; Noga et al., 1988), and the lateral funiculus (Yamaguchi, 1986), it appears that there is a fair degree of overlap within these areas. Likewise, it is difficult to make any conclusive statement concerning a topographic relationship for the MLR descending pathways to forelimb and hindlimb spinal locomotor centers from data obtained in the present experiments. In all but one animal (Fig. $3 B$ ), stimulation of the MLR produced quadrupedal locomotion, indicating that the stimulating electrode was positioned appropriately to activate all spinal locomotor centers. Interruption of the dorsal aspect of the spinal cord had no observable effect on forelimb or hindlimb locomotion produced by stimulation of the MLR in most experiments (Figs. 1, $3 A, B, D, F, I$ ). This is in agreement with the original observation that the VLF is required for quadrupedal locomotion induced by stimulation of the MLR (Steeves and Jordan, 1980). While some decrement in the quality of locomotion produced by stimulation of the MLR was observed for 3 of 12 stimulation sites following lesions of the dorsal aspect of the spinal cord, this decrement could be due to a general deterioration of the preparation following the lesioning procedure, rather than to the interruption of some component of the descending locomotor pathway. Alternative$\mathrm{ly}$, the forelimb locomotor deficits observed following a lesion that included part of the DLF in one experiment (Fig. $3 H$ ) may be due to a partial interruption of a more dorsally located projection mediating forelimb locomotion. This view has been proposed by Yamaguchi (1986), who stated that the DLF may mediate some of the forelimb locomotion produced by stimulation of the MLR (though the VLF may also contribute to the production of forelimb locomotion).

As summarized in the introductory remarks, the evidence used to conclude that the MLR projects to the spinal cord via the PLR-DLF pathway was based primarily on electrophysio- 
logical experiments. For example, stimulation of the region between the MLR and the PLR may produce locomotion (Mori et al., 1977), thus giving the appearance of continuity between the two structures. However, evidence has been provided that a region medial to the MLR site used in the present experiments may also produce locomotion when stimulated (Garcia-Rill et al., 1983; Shefchyk et al., 1984). This medial site has been shown to project laterally through the lateral tegmentum (Garcia-Rill et al., 1983; see also Shefchyk et al., 1984) and may represent an afferent pathway to the spinal nucleus of the trigeminal nerve (Noga et al., 1988). The results reported by Mori et al. (1977) may thus be due to stimulation of the laterally directed fibers projecting from this medial site to the PLR region. This projection would overlap the medially directed projections of the MLR on their course to the MedRF (Steeves and Jordan, 1984). This explanation could also account for the results obtained by Shik and Yagodnitsyn (1978), who demonstrated that stimulation of areas caudal to the MLR could activate cells within the PLR.

Other evidence used to conclude that the MLR projects to the spinal cord via the PLR-DLF pathway was the observation that stimulation of the PLR (at strengths subthreshold for the initiation of locomotion) could facilitate locomotion induced by stimulation of the MLR (Mori et al., 1977, 1978a). However, this may simply reflect the fact that the PLR may project to the MedRF (Selionov and Shik, 1981, 1984; see The PLR descending pathways, below) and converge on reticulospinal cells also activated by stimuli applied in the MLR. The fact that the MLR and PLR converge onto propriospinal cells in the $\mathrm{C} 2-\mathrm{C} 3$ spinal segments has also been used as evidence for the suggestion that the MLR projects via the PLR (Kazennikov et al., 1983a, 1985). However, removal of these cells by extended dorsal hemisections does not abolish MLR-evoked locomotion (or affect the electrical threshold) and demonstrates that their function is not necessary for the production of locomotion by MLR stimulation. Shik (1983) has also suggested that the field potentials recorded in the MLR from stimulation of the PLR (Mori et al., 1977) may represent ascending fibers from the PLR and that the MLR may therefore be part of an ascending system. Stimulation of the MLR might then evoke locomotion due to antidromic excitation of the PLR. This hypothesis is not supported by the observation that isolation of the dorsolateral tegmentum did not abolish the ability of the MLR to produce quadrupedal locomotion when electrically stimulated.

\section{The PLR descending pathways}

The results of the present study demonstrate that the PLR can produce locomotion by activation of cells in the MedRF and through a pathway in the ventral half of the spinal cord (cf. Fig. 7). It is suggested that the PLR converges onto cells in the MedRF that mediate locomotion produced by stimulation of the MLR. These conclusions are based on the observations that (1) cooling of the MedRF (in an area that also blocks locomotion produced by stimulation of the MLR) can abolish the locomotion produced by stimulation of the PLR, (2) stimulation of the PLR may produce forelimb or hindlimb locomotion following extended spinal cord dorsal hemisections, and (3) cooling of the ipsilateral VLF can reversibly abolish PLR-evoked locomotion. Activation of cells within the lateral tegmentum may thus initiate locomotion by gaining access to descending motor pathways originating within the MedRF.

The observation that locomotion may be produced by a path-

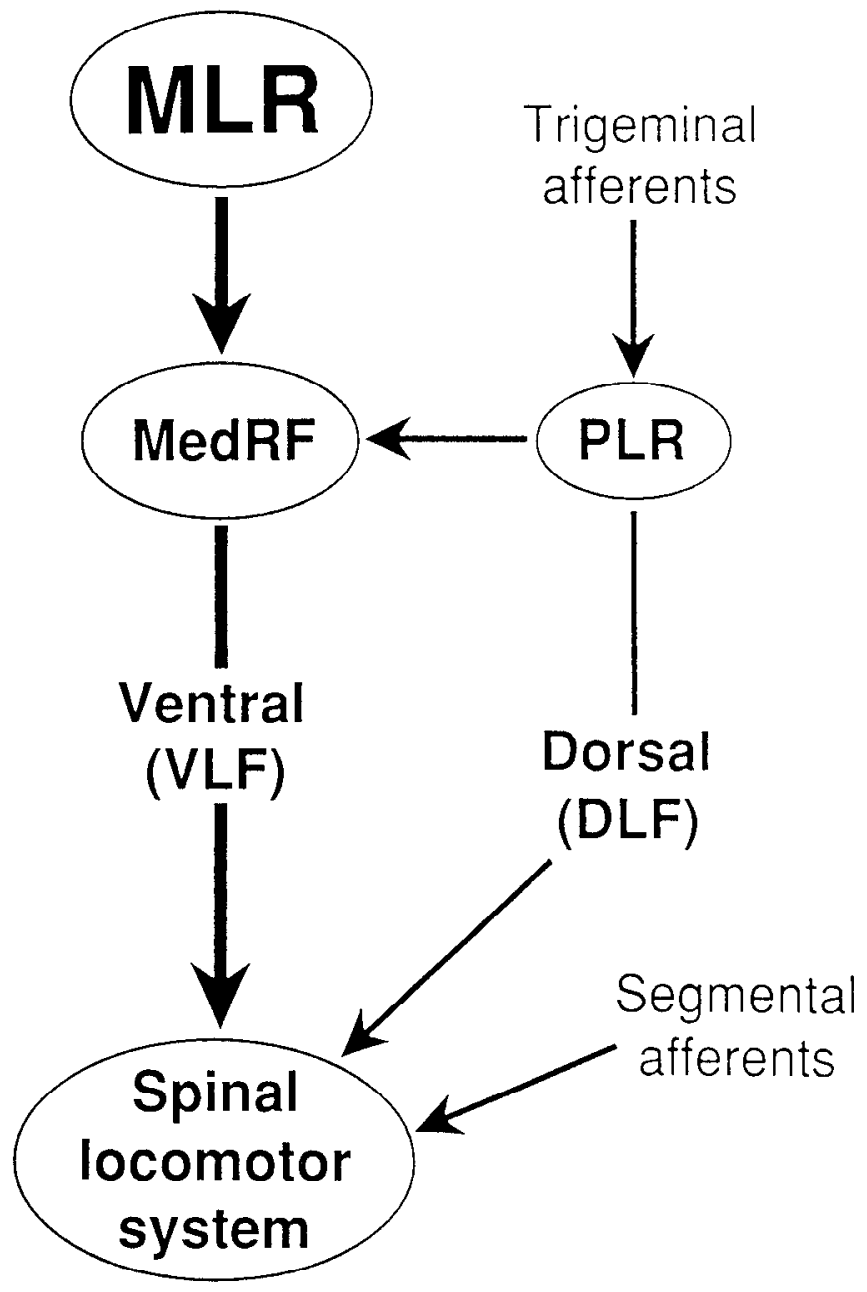

Figure 7. Organization and functional interactions of locomotor systems within the brainstem and their descending trajectories to spinal locomotor centers located in the cervical and lumbar enlargements. Relay sites in the pathways are circled.

way that projects to the MedRF and through the VLF is in contrast to the hypothesis that locomotion produced by stimulation of the PLR is mediated by a polysynaptic pathway that descends in the lateral tegmentum of the brainstem and continues in the spinal cord in the DLF (Kazennikov et al., 1979, 1980, 1983a,b, 1985; Selionov and Shik, 1981, 1984; Shik, 1983). This hypothesis was based primarily on the report that destruction of the dorsal horn (cclls of origin of the pathway) at the C2-C3 level (Kazennikov et al., 1980) for distances of 4$6 \mathrm{~mm}$ was required to abolish PLR-evoked locomotion if the lateral and ventral funiculi remained intact, whereas local (rostrocaudal length, $1-1.5 \mathrm{~mm}$ ) lesions had no effect. It was thought that activity in the propriospinal pathway could bypass the local lesions (c.f. Budakova and Shik, 1980) because the characteristic length of axons in the polysynaptic pathway is $3-5 \mathrm{~mm}$ at this level (Kazennikov et al., 1979). While it may be true that the PLR may project via this pathway (see below), it is certainly not the only route of activation because stimulation of the PLR may produce locomotion following lesions that destroy the spinal gray or the dorsal half of the spinal cord for up to $8.5 \mathrm{~mm}$. The reasons for the loss of locomotor capability of the animals in the study by Kazennikov et al. (1979) are not clear. One possibility is that the threshold for activation of the PLR-MedRF 
pathway was outside of the tested range of stimulation. Alternatively, the loss of locomotor capability was due either to damage of the MedRF-VLF pathway when the spinal cord was lesioned or to a general deterioration in the health of the animal.

The results of the present study also indicate that PLR stimulation may produce locomotion by activation of a pathway that descends through the lateral tegmentum of the brainstem and through the dorsal half of the spinal cord (Fig. 7). This conclusion is based on the following observations: (1) stimulation of the PLR may produce locomotion following isolation of the lateral tegmentum of the brainstem from the MedRF and the MLR, and (2) loss of PLR-evoked locomotion may occur following transections of the DLF or extended dorsal hemisections of the spinal cord. While it is difficult to exclude the possibility that the loss of PLR-evoked locomotion following these extensive lesions is due to a general deterioration of the preparation, the fact that stimulation of the MLR could still produce locomotion in many of these experiments suggests otherwise. Similar results have been reported for the Atlantic stingray by Livingston and Leonard (1990), who showed that swimming may be obtained by stimulation of lateral tegmental areas following lesions that isolate it from the MedRF. In this context, these data support the proposal that stimulation of the PLR may activate a pathway that descends to the spinal locomotor centers via the dorsal aspect of the spinal cord (Kazennikov et al., 1979, 1980, 1983a,b; Selionov and Shik, 1981; Shik, 1983).

Stimulation of the PLR may not necessarily produce quadrupedal locomotion at all times (like that seen with MLR stimulation), and bipedal (forelimb or hindlimb) or other combinations may be observed (Shik and Yagodnitsyn, 1977, 1978; Mori et al., 1978a,b; Noga et al., 1988). While some evidence was presented suggesting that a topographic organization may be present for locomotion-producing sites within the PLR (Ross and Sinnamon, 1984; Noga et al., 1988), it is not certain whether a topographic relationship exists for the descending pathways originating from this area. Although forelimb locomotion was affected slightly more often than hindlimb locomotion by lesions of the dorsal aspect of the spinal cord, stimulation of the PLR may also elicit forelimb locomotion via activation of a pathway that descends through the ventral half of the spinal cord (Figs. $3 C, 4,6)$. It is therefore difficult to make any conclusive statement concerning a topographic scheme for descending pathways activated by electrical stimulation applied to the PLR with the data obtained in the present study. Many possible factors (e.g., the state of excitability of the cells interposed within the pathway, the position of the stimulating electrode, etc.) may influence the relative effectiveness of one pathway or another in mediating the locomotor behavior produced with stimulation of the PLR. Further elaboration on these factors would require a more detailed study that would be aided by the use of neurochemical microinjection techniques.

The observation that stimulation of the PLR may produce forelimb and/or hindlimb locomotion by activation of pathways that project through either dorsal or ventral spinal cord is consistent with the view that the PLR is part of the trigeminal system involved in sensory activation of locomotion (Noga et al., 1988). This is also consistent with anatomical studies that demonstrate the presence of trigeminoreticular (Nauta and Kuypers, 1958; Stewart and King, 1963) and intranuclear trigeminospinal pathways (Matsushita et al., 1981, 1982). In this way, sensory activation of locomotion (Aoki and Mori, 1981; Bayev et al., 1988; Beresovskii and Bayev, 1988; Noga et al., 1988) may be achieved via a connection between the PLR and the reticulospinal system (by activation of cells in the MedRF that project via the VLF) or the propriospinal system (by activation of cells in the lateral tegmentum and possibly dorsal horn that project via the DLF). These observations are also supported by the observation that trigeminal peripheral receptive ficld stimulation produced locomotion when glutamic acid was injected into the MedRF (Noga et al., 1988). Thus, the activation of cells in the MedRF by stimulation of either the PLR (Selionov and Shik, 1981, 1984; Mori et al., 1986) or trigeminal afferents (Lamarche et al., 1960; Noga et al., 1988) may be mediated by similar pathways. Whether the propriospinal system proposed to mediate the effects of PLR stimulation (Kazennikov et al., 1979, 1980, 1983a,b, 1985; Selionov and Shik, 1981, 1984; Shik, 1983) is equivalent to the pathway revealed in this study or the pathway described by Matsushita et al. (1981, 1982) remains to be shown. If this were so, one would expect that the cells of origin of the propriospinal pathway activated by stimuli applied to the PLR (Kazennikov et al., 1979, 1983a,b; Shik, 1983) should be capable of producing locomotion with injections of neuroactive substances and should be responsive to stimulation of the trigeminal peripheral field.

\section{The organization of brainstem and spinal cord locomotor pathways}

The pathways in the mammalian brainstem and spinal cord involved in the production of locomotion in the decerebrate animal are summarized in Figure 7. Parallel pathways that may also be activated during locomotion in this preparation (cf. Armstrong, 1988) are excluded for the sake of brevity. Briefly, the MLR produces locomotion by activation of cells within the MedRF, which then project to the spinal locomotor system via the ventral aspect of the spinal cord (VLF). In contrast, cells within the lateral tegmentum of the brainstem (the PLR) that receive trigeminal afferent input from the mesencephalic nucleus of the trigeminal nerve and from the trigeminal peripheral nerve (Noga et al., 1988) may activate locomotor systems either by a convergence onto the motor pathway in the MedRF or by a pathway that continues in the lateral tegmentum and the dorsal aspect of the spinal cord. In terms of locomotor system organization, the pathway found at all levels of the dorsal aspect of the spinal cord that can produce locomotor movements when stimulated (Lennard and Stein, 1977; Jacobson and Hollyday, 1982; Kazennikov et al., 1983b, 1985; Williams et al., 1984; Yamaguchi, 1986, 1987) may be regarded as part of the propriospinal system that integrates sensory afferent information and that eventually converges on the spinal locomotor system. Evidence in favor of this interpretation comes from the observation of Shimamura et al. (1984), who found that bilateral lesions of the DLF could abolish locomotion produced by stimulation of joint afferents in the thalamic cat. We propose that the PLR of the brainstem is a continuation of this propriospinal system. In this view, the trigeminal afferents that converge on the PLR may be regarded as synonymous with the segmental afferents that induce locomotion in other preparations (Sherrington, 1910, 1913; Jankowska et al., 1967; Budakova, 1972; Grillner and Zangger, 1979; Shimamura et al., 1984).

\section{References}

Afelt Z (1974) Functional significance of ventral descending tracts of the spinal cord in the cat. Acta Neurobiol Exp 34:393-407.

Afelt Z, Kasicki S, Sybirska E, Zagrodzka J (1975) Limb coordinations 
during locomotion in amygdalar, rubral and funicular cats. Acta Neurobiol Exp 35:379-388.

Amemiya M, Yamaguchi T (1984) Fictive locomotion of the forelimb evoked by stimulation of the mesencephalic locomotor region in the decerebrate cat. Neurosci Lett 50:91-96.

Aoki M, Mori S (1981) Locomotion elicited by pinna stimulation in the acutc precollicular-post-mammillary decerebrate cat. Brain Res 214:424-428.

Armstrong DM (1988) The supraspinal control of mammalian locomotion. J Physiol (Lond) 405:1-37.

Bayev KV, Beresovskii VK, Kebkalo TG, Savoskina LA (1988) Afferent and efferent connections of brainstem locomotor regions: study by means of horseradish peroxidase transport technique. Neuroscience 26:871-891.

Beresovskii VK, Bayev KV (1988) New locomotor regions of the brainstem revealed by means of electrical stimulation. Neuroscience 26:863-869.

Berman AL (1968) The brain stem of the cat. Madison: University of Wisconsin.

Brooks VB (1983) Study of brain function by local, reversible cooling. Rev Physiol Biochem Pharmacol 95:1-109.

Budakova NN (1972) Stepping movements evoked by repetitive dorsal root stimulation in a mesencephalic cat. Neurosci Behav Physiol 5:355-363.

Budakova NN, Shik ML (1980) Continuity of the bulbar locomotor strip is not essential for walking. Bull Exp Biol Med 89:1-4.

Drew T, Rossignol S (1984) Phase-dependent responses evoked in limb muscles by stimulation of medullary reticular formation during locomotion in thalamic cats. J Neurophysiol 52:653-675.

Eidelberg E (1980) Locomotor control in macaque monkeys. In: Advances in Physiological Sciences, Vol 1, Regulatory functions of the CNS. Motion and organization principles (Szentágothai J, Palkovits M, Hámori J, eds), pp 187-188. Budapest: Pergamon. Akadémiai Kiadó.

Eidelberg E (1981) Consequences of spinal cord lesions upon motor function, with special reference to locomotor activity. Prog Neurobiol 17:185-202.

Eidelberg E, Story JL, Walden JG, Meyer BL (1981a) Anatomical correlates of return of locomotor function after partial spinal cord lesions in cats. Exp Brain Res 42:81-88.

Eidelberg E, Walden JG, Nguyen LH (1981b) Locomotor control in macaque monkeys. Brain 104:647-663.

Garcia-Rill E (1986) The basal ganglia and the locomotor regions. Brain Res Rev 11:47-63.

Garcia-Rill E, Skinner RD (1987) The mesencephalic locomotor region. II. Projections to reticulospinal neurons. Brain Res 411:13-20.

Garcia-Rill E, Skinner RD, Gilmore SA, Owings R (1983) Connections of the mesencephalic locomotor region (MLR). II. Afferents and efferents. Brain Res Bull 10:63-71.

Grillner S, Zangger $P$ (1979) On the central generation of locomotion in the low spinal cat. Exp Brain Res 34:241-261.

Jacobson RD, Hollyday M (1982) Electrically evoked walking and fictive locomotion in the chick. J Neurophysiol 48:257-270.

Jankowska E, Jukes MGM, Lund S, Lundberg A (1967) The effect of DOPA on the spinal cord. VI. Half-centre organization of interneurones transmitting effects from the flexor reflex afferents. Acta Physiol Scand 70:389-402.

Jordan LM (1986) Initiation of locomotion from the mammalian brainstem. In: Wenner-Gren Centre International Symposium Series, Vol 45, Neurobiology of vertebrate locomotion (Grillner S, Stein PSG, Stuart DG, Forssberg H, Herman RM, eds), pp 21-37. London: Macmillan.

Kazennikov OV, Selionov VA, Shik ML, Yakovleva GV (1979) Neurons of upper cervical segments responding to stimulation of the bulbar "locomotor strip." Neurophysiology 11:179-185.

Kazennikov OV, Shik ML, Yakovleva GV (1980) Two pathways for the brainstem "locomotor influence" on the spinal cord. Fiziol Zh SSSR 66:1260-1263.

Kazennikov OV, Shik ML, Yakovleva GV (1983a) Responses of upper cervical spinal neurons in cats to stimulation of the brain-stem locomotor region at different frequencies. Neurophysiology 15:256261.

Kazennikov OV, Shik ML, Yakovleva GV (1983b) Stepping movements induced in cats by stimulation of the dorsolateral funiculus of the spinal cord. Bull Exp Biol Med 96:1036-1039.

Kazennikov OV, Shik ML, Yakovleva GV (1985) Synaptic responses of propriospinal neurons to stimulation of the stepping strip of the dorsolateral funiculus in cats. Neurophysiology 17:195-202.

Klüver H, Barrera E (1953) A method for the combined staining of cells and fibres in the nervous system. J Neuropath Exp Neurol 12: 400-403.

Lamarche G, Langlois JM, Héon M (1960) Unit study of the trigeminal projections in the reticular formation of the medulla oblongata in the cat. Can J Biochem Physiol 38:1163-1166.

Lennard PR, Stein PSG (1977) Swimming movements elicited by electrical stimulation of turtle spinal cord. I. Low spinal and intact preparations. J Neurophysiol 40:768-778.

Livingston CA, Leonard RB (1990) Locomotion evoked by stimulation of the brain stem in the Atlantic stingray, Dasyatis sabina. $\mathrm{J}$ Neurosci 10:194-204.

Matsushita M, Okado N, Ikeda M. Hosoya Y (1981) Descending projections from the spinal and mesencephalic nuclei of the trigeminal nerve to the spinal cord in the cat. A study with the horseradish peroxidase technique. J Comp Neurol 196:173-187.

Matsushita M, Ikeda M, Okado N (1982) The cells of origin of the trigeminothalamic, trigeminospinal, and trigeminocerebellar projections in the cat. Neuroscience 7:1439-1454.

Mori S, Shik ML, Yagodnitsyn AS (1977) Role of pontine tegmentum for locomotor control in mesencephalic cat. J Neurophysiol 40:284295.

Mori S, Nishimura H, Kurakami C, Yamamura T, Aoki M (1978a) Controlled locomotion in the mesencephalic cat: distribution of facilitatory and inhibitory regions within pontine tegmentum. J Neurophysiol 41:1580-1591.

Mori S, Nishimura H, Kurakami C, Yamamura T, Aoki M (1978b) Lower brain-stem "locomotor region" in the mesencephalic cat. In: Integrative control functions of the brain, Vol 1 (Ito M, ed), pp 209 211. New York: Elsevier/North Holland.

Mori S, Kawahara K, Sakamoto T (1982) Supraspinal aspects of locomotion in the mesencephalic cat. In: Symposia of the society for cxpcrimental biology, No 37, Neural origin of rhythmic movements (Roberts A, Roberts BL, eds), pp 445-468. New York: Cambridge UP.

Mori S, Selionov VA, Shik ML (1986) Responses of bulbar neurons to stimulation of locomotor and inhibitory sites in the cat brainstem. Neurophysiology 18:386-393.

Nauta WJH, Kuypers HGJM (1958) Some ascending pathways in the brainstem reticular formation. In: Reticular formation of the brainstem (Jasper HH, Proctor LD, Knighton RS, Noshay WC, Costello RT, eds), pp 3-30. Boston: Little, Brown.

Noga BR (1988) An investigation of descending brainstem pathways involved in the production of locomotion. $\mathrm{PhD}$ thesis, University of Manitoba.

Noga BR, Kriellaars DJ, Brownstone RM, Mallory B, Jordan LM (1986) A common pathway for MLR evoked PSPs and the initiation of locomotion. Soc Neurosci Abstr 12:877.

Noga BR, Kettler J, Jordan LM (1988) Locomotion produced in mesencephalic cats by injections of putative transmitter substances and antagonists into the medial reticular formation and the pontomedullary locomotor strip. J Neurosci 8:2074-2086.

Nord SG, Ross GS (1973) Responses of trigeminal units in the monkey bulbar lateral reticular formation to noxious and non-noxious stimulation of the face: experimental and theoretical considerations. Brain Res 58:385-399.

Orlovsky GN (1969) Electrical activity in the brainstem and descending pathways in guided locomotion. Fiziol Zh SSSR 55:437-444.

Orlovsky GN (1970) Connexions of the reticulo-spinal neurons with the "locomotor sections" of the brainstem. Biophysics 15:178-186.

Ross GS, Sinnamon IIM (1984) Forelimb and hindlimb stepping by the anesthetized rat elicited by electrical stimulation of the pons and medulla. Physiol Behav 33:201-208.

Selionov VA, Shik ML (1981) Responses of medullary neurons to microstimulation of the "locomotor strip" in cats. Neurophysiology 13:199-205.

Selionov VA, Shik ML (1984) Medullary locomotor strip and column in the cat. Neuroscience 13:1267-1278.

Shefchyk SJ, Jell RM, Jordan LM (1984) Reversible cooling of the brainstem reveals areas required for mesencephalic locomotor region evoked treadmill locomotion. Exp Brain Res 56:257-262.

Sherrington CS (1910) Flexion-reflex of the limb, crossed extension reflex, and reflex stepping and standing. J Physiol (Lond) 40:28-121.

Sherrington CS (1913) Further observations on the production of reflex 
stepping by combination of reflex excitation with reflex inhibition. J Physiol (Lond) 47:196-214.

Shik ML (1983) Action of the brainstem locomotor region on spinal stepping generators via propriospinal pathways. In: Spinal cord reconstruction (Kao CC, Bunge RP, Reier PJ, eds), pp 421-434. New York: Raven.

Shik ML, Yagodnitsyn AS (1977) The pontobulbar "locomotor strip." Neurophysiology 9:72-74.

Shik ML, Yagodnitsyn AS (1978) Unit responses in the "locomotor strip" of the cat hindbrain to microstimulation. Neurophysiology 10 : 373-379.

Shik ML, Severin FV, Orlovsky GN (1966) Control of walking and running by means of electrical stimulation of the mid-brain. Biophysics $4: 756-765$.

Shik ML, Severin FV, Orlovsky GN (1967) Structures of the brain stem responsible for evoked locomotion. Fiziol Zh SSSR 53:11251132.

Shik ML, Orlovsky GN, Severin FV (1968) Locomotion of the mesencephalic cat elicited by stimulation of the pyramids. Biophysics 13: 143-152.

Shimamura M, Kogure I, Fuwa T (1984) Role of joint afferents in relation to the initiation of forelimb stepping in thalamic cats. Brain Res 297:225-234.
Sholomenko GN, Steeves JD (1987) Effects of selective spinal cord lesions on hind limb locomotion in birds. Exp Neurol 95:403-418.

Steeves JD, Jordan LM (1980) Localization of a descending pathway in the spinal cord which is necessary for controlled treadmill locomotion. Neurosci Lett 20:283-288.

Steeves JD, Jordan LM (1984) Autoradiographic demonstration of the projections from the mesencephalic locomotor region. Brain Res 307:263-276.

Stewart WA, King RB (1963) Fiber projections from the nucleus caudalis of the spinal trigeminal nucleus. J Comp Neurol 121:271-286.

Torvik $\Lambda$ (1956) $\Lambda$ fferent connections to the sensory trigeminal nuclei, the nucleus of the solitary tract and adjacent structures. J Comp Neurol 106:51-141.

Williams BJ, Livingston CA, Leonard RB (1984) Spinal cord pathways involved in initiation of swimming in the stingray, Dasyatis sabina: spinal cord stimulation and lesions. J Neurophysiol 51:578-591.

Yamaguchi $T$ (1986) Descending pathways eliciting forelimb stepping in the lateral funiculus: experimental studies with stimulation and lesions of the cervical cord in decerebrate cats. Brain Res 379:125136.

Yamaguchi T (1987) Monopodal fictive locomotion evoked by cervical cord stimulation in decerebrate cats. Neurosci Lett 74:69-74. 